\title{
Primary Retinal Vasculitis Vs Eales' Disease
}

\author{
Courtney M. Crawford ${ }^{1 *}$, Yihharn Hwang ${ }^{2}$ \\ ${ }^{1}$ North Texas Retina Consultants, Fort Worth TX \\ ${ }^{2}$ Ophthalmic Consultants of Boston, Boston MA
}

Received: 12 September, 2016; Accepted: 20 September, 2016; Published: 30 September, 2016

*Corresponding author: Courtney Crawford, M.D, North Texas Retina Consultants, 101 Chuckwagon Trail, Fort Worth TX 76087, Tel: 817-441-1212; Fax: 817-441-2851; Email: courtneymcrawford@gmail.com

\begin{abstract}
Purpose: To report on a case of a patient with primary retinal vasculitis.

Methods: Retrospective single case report.

Patient: A 27-year-old female presented with decreased vision in her left eye for the past year. On exam, her visual acuity for the left eye was $20 / 50$. She has had trouble accessing medical care but denies any illness, infections or trauma.

Results: Upon ophthalmic examination, retinal abnormalities were seen such as the presence of peripheral vessel sheathing, peripheral non-perfusion and extensive neovascularization. Fluorescein angiography revealed significant late leakage of the retina vessels with greater severity in the left eye. Laboratory findings were unremarkable. After diagnosis of primary retinal vasculitis, the patient was treated with photocoagulation in both eyes. She also received two treatments of intravitreal bevacizumab for the neovascularization.

Conclusion: Primary retinal vasculitis is often hard to diagnose due to disease course variation. The cause of retinal vasculitis must first be established as either an isolated condition or due to an inflammatory systemic condition. A thorough review of the patient's medical history, review of systems, ophthalmic and physical examination, along with proper laboratory tests must be performed in order to ensure accurate diagnosis and treatment.
\end{abstract}

Keywords: Primary retinal vasculitis; Eales' disease; Perivascular sheathing,; Vascular leakage; Neovascularization

\section{Introduction}

Retinal vasculitis is a poorly characterized, potentially sightthreatening, inflammatory ocular condition that occurs when there is the presence of abnormal blood vessels in the retina. The annual incidence of retinal vasculitis in the United States is estimated at 1-2 per 100,000 with variation between regions. Because it is not well understood, the definition of "retinal vasculitis" is not well established. Currently, the Standardization of Uveitis Nomenclature Working Group considers "perivascular sheathing and vascular leakage/occlusion on fluorescein angiogram as evidence of retinal vascular disease" in classifying retinal vasculitis [2]. There are two main causes of retina vasculitis: primary ocular vasculitis, occurring as an isolated condition or secondary retinal vasculitis, caused by an inflammatory systemic condition. Common systemic diseases associated with retinal vasculitis include Behcet's disease, sarcoidosis, multiple sclerosis, systemic lupus erythematosus, Wegener granulomatosis, polyarteritis nodosa and other rheumatologic conditions. Infectious agents may also cause retinal vasculitis such as syphilis, tuberculosis, and Lyme disease. Common diseases associated with isolated conditions of retinal vasculitis include primary retinal vasculitis, Eales' disease, pars planitis, birdshot retinochoroidopathy, and Fuchs uveitis syndrome. Many autoimmune manifestations have been seen with retinal vasculitis when no systemic diseases are present; however, the exact etiology is unclear $[1,2]$.

Clinically, patients with retinal vasculitis most commonly present with blurred vision, altered color perception, distortion of images, floaters and scotomas. However, sometimes patients may present with no visual symptoms. Retinal vasculitis can be detected using ophthalmologic examinations and fluorescein angiography, which can show perivascular sheathing, vascular leakage, inflammatory cells of the vitreous body, narrowing of the retinal blood vessels, and neovascularization. Late leakage or staining of retinal vessels is also evident on FA. Sometimes, mild anterior or posterior uveitis may be seen. The presence of choroidal inflammation may indicate sarcoidosis or birdshot retinochoroidopathy $[1,6]$ Active vasculitis causes the formation of exudates around retinal vessels, which results in white sheathing or cuffing of the vasculature. This can lead to vascular leakage, which causes retinal swelling and macular edema that can greatly affect visual acuity and lead to vision loss. Visual acuity prognosis is difficult to anticipate due to complications such as macular ischemia, branch and central retinal vein occlusion, persistent neovascularization and vitreous hemorrhages.

Secondary retinal vasculitis due to systemic diseases more often leads to retinal occlusion, rather than vascular sheathing. The retinal arterioles or branch retinal arteries can become occluded by microthrombosis without significant intraocular inflammation, a difference from active vasculitis $[2,3]$.

\section{Case}

A 27-year old female from Saudi Arabia presents with decreased vision in her left eye for a year. She denies any recent 
illnesses, infections or trauma and is currently on no medications. She states that she has had difficulty accessing medical care. A negative review of systems is unremarkable for cough, fever, night sweats, joint pain or orogenital ulcerations.

On exam, her vision was $20 / 20$ OD and 20/50 OS with symmetrically reactive pupils and normal intraocular pressures. Anterior segment exam was normal. However, posterior segment exam of the right eye showed peripheral vessel sheathing with peripheral non-perfusion. The left eye showed extensive neovascularization of the optic disc, central and peripheral non-perfusion, along with intraretinal microvascular anomalies (Figure 1). Fluorescein angiography of both eyes revealed significant late leakage of the retina vessels with the left eye to a greater degree (Figure 2b). Spectral Domain OCT showed appropriate foveal contour with no macular edema or subretinal fluid. The following laboratory tests were performed: CBC with peripheral smear, PPD, Quantiferon, ACE, lysozyme, ESR, RPR, ANA, and a chest $x$-ray. All findings were negative. The patient was also found to be negative for diabetes, hypertension, HIV and TB.

\section{Discussion}

In diagnosing primary retinal vasculitis, there should be a differential diagnosis based on a detailed history, review of systems, ophthalmic and physical examination. If the patient's workup suggests an underlying systemic cause, then further examinations and laboratory tests should be performed. The absence of any diagnostic clues from the workup and a negative

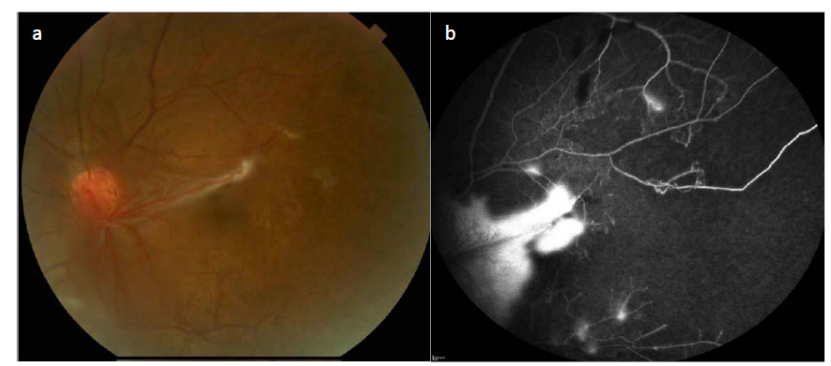

Figure 1: (a) A color fundus photograph shows presence of intraretinal microvascular anomalies, central and peripheral non-perfusion and extensive neovascularization of the optic disc. (b) A fluorescein angiogram that shows retinal vascular leakage.

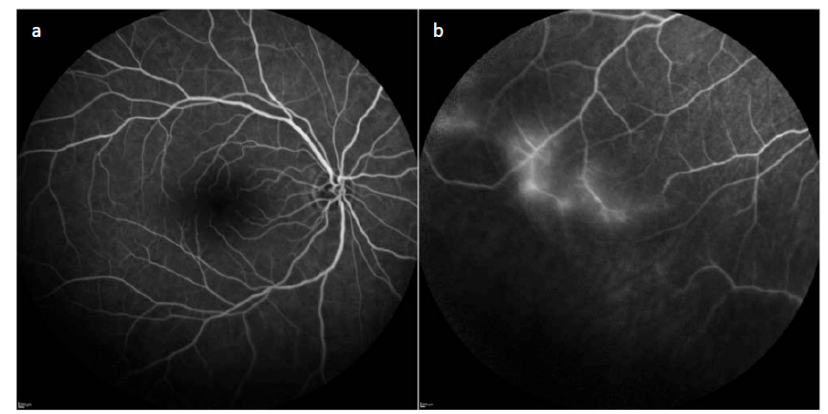

Figure 2: (a) Posterior pole is unremarkable. (b) Late leakage and nonperfusion of periphery medical history for systemic diseases associated with retinal vasculitis would suggest primary retinal vasculitis. However, the pathophysiology of primary retinal vasculitis is often closely aligned with Eales' disease. Eales' disease is also associated with isolated conditions of retinal vasculitis, most often seen in young men. Characteristic features include inflammation of the peripheral retinal vasculature causing retinal phlebitis that can lead to retinal non-perfusion, neovascularization and recurrent vitreous hemorrhages. It is also highly associated with individuals with tuberculosis. The natural progression of Eales' disease begins with inflammation, leading to occlusion and then lastly, the presence of neovascularization. Other clinical presentations seen in Eales' disease include exudative vasculitis, cotton-wool spots, macular edema, and anterior uveitis. Although the differentiation between primary retinal vasculitis and Eales' disease is often unclear, there are certain distinctions seen in each of the diseases. The patient was ultimately diagnosed with primary retinal vasculitis due to the location of her abnormal retinal vasculature being more posterior, sectorial and exudative. Disease presentation is also seen equally in both genders while in Eales' disease, it is more prominent in males. The patient also exhibited peripheral sheathing and nonperfusion in both eyes. In individuals with Eales' disease, peripheral vascular inflammation only occurs at a minimum while retinal vascular occlusions are more common $[6,7]$. Eales' disease is also often associated with positive TB patients, which was not seen in this case. Nevertheless, the diagnostic workup and treatment for primary retinal vasculitis and Eales' disease are similar.

Due to disease course variation, the poor outcome of primary retinal vasculitis is dependent on a number of factors. To control inflammation of the retinal vasculature, the main treatment option for primary retinal vasculitis often involves the use of corticosteroids with or without immunosuppressive therapy. Cyclosporine and azathioprine have shown to be effective in reducing inflammation. Anti-tuberculosis treatment is given for positive TB patients, which is more often seen in individuals with Eales' disease. Laser photocoagulation is often combined with bevacizumab intravitreal injections to treat neovascularization and prevent further retinal ischemia [2, 4]. Our patient responded well to panretinal photocoagulation of the left eye and scatter photocoagulation of the right eye. Additionally, the extensive neovascularization of the disc regressed with two treatments intravitreal bevacizumab over a 6 -month period. The prognosis of patients with primary retinal vasculitis is variable due to the heterogenic nature of the disease. Some patients that undergo treatment have full preservation of their ocular function and vision. However, others may have permanent loss of function despite treatment. Current status of patient is unknown. Due to her return to Saudi Arabia, she has been lost to follow-up.

\section{References}

1. Walton RC, Ashmore ED. Retinal vasculitis. Curr Opin Ophthalmol. 2003;14(6):413-9.

2. Ku JH, Ali A, Suhler EB, Choi D, Rosenbaum JT. Characteristics and Visual Outcome of Patients with Retinal Vasculitis. Arch Ophthalmol. 2012;130(10):1261-6. 
3. El-Asrar AMA, Herbort CP, Tabbara KF. Differential Diagnosis of Retinal Vasculitis. Middle East Afr J Ophthalmol. 2009;16(4):202-18. doi: 10.4103/0974-9233.58423.

4. Talat L, Lightman S, Tomkins-Netzer O. Ischemic Retinal Vasculitis and Its Management. J Ophthalmol. 2014;2014:197675. doi: 10.1155/2014/197675.

5. Biswas J, Ravi RK, Narayanasamy A, Kulandai LT, Madhavan HN Eales' disease - current concepts in diagnosis and management. J
Ophthalmic Inflamm Infect. 2013;3(1):11. doi: 10.1186/1869-57603-11.

6. Ryan SJ, Schachat AP, Wilkinson CP, Hinton DR, Sadda SR, et al. Retina. 2013;5(2):1479-1484.

7. George RK, Walton RC, Whitcup SM, Nussenblatt RB. Primary retinal vasculitis. Systemic associations and diagnostic evaluation. Ophthalmology. 1996;103(3):384-389. 